CERN-TH/98-55

$\mathrm{IFT} / 3 / 98$

\title{
Higher-order QED corrections to $e^{+} e^{-} \rightarrow \nu \bar{\nu} \gamma$ at LEP2
}

\author{
A. Jachołkowska ${ }^{1}$, J. Kalinowski ${ }^{2}$ and Z. Wa̧s ${ }^{3,4}$ \\ ${ }^{1}$ Laboratoire de l'Accélérateur Lineaire,CNRS-IN2P3, 914050 ORSAY, France \\ ${ }^{2}$ Institute of Theoretical Physics, Hoża 69, 00681 Warsaw, Poland \\ ${ }^{3}$ CERN, Theory Division, CH-1211 Geneva 23, Switzerland \\ ${ }^{4}$ Institute of Nuclear Physics, ul. Kawiory 26a, Cracow, Poland
}

\begin{abstract}
The process $e^{+} e^{-} \rightarrow \nu \bar{\nu}+\gamma$ with a distinctive "photon-plus-missing-energy" signal can serve as an important tool to search for new physics at LEP2. It can be exploited to measure the $W W \gamma$ coupling, or to search for weakly interacting and invisible (s)particles. For meaningful comparisons of experimental data with theoretical predictions, higher-order QED corrections due to multiphoton emission must be taken into account. In the present paper we explain how the $W W \gamma$ coupling has been incorporated into the KORALZ Monte Carlo program, which can now be used for simulations of $e^{+} e^{-} \rightarrow \nu \bar{\nu}+\gamma$ events with higher-order QED corrections. The strategy of how to perform an experimental analysis in the presence of experimental cuts is proposed. The question of systematic uncertainties is addressed and some numerical results for the phenomenologically interesting case of anomalous $W W \gamma$ couplings are also given.
\end{abstract}

CERN-TH/98-55

February 1998 


\section{Introduction}

One of the main goals of experiments at high energy consists in comparing new and so far unexplored measurable quantities with the Standard Model (SM) predictions and thus providing its important tests in a new experimental domain. On the other hand, any discrepancy can be a sign of new physics. From the practical point of view it is thus important to confront new experimental data with theoretical predictions for observables which are: (i) sensitive to expected new physics, (ii) under control of systematic uncertainties for the data and theoretical predictions, and (iii) have high statistics. To optimize the experimental analysis and to suppress SM backgrounds, complicated patterns of kinematical cuts are often introduced. One should also bear in mind that the detector sensitivity varies over detector acceptance in a complicated manner. That is why high-precision physics Monte Carlo programs, including complete Standard Model predictions as well as contributions from possible 'new physics', are useful and in some cases even mandatory for such projects [1].

The measurement of the nature of three-gauge-boson couplings has been identified as one of the most important topics in the LEP2 scientific program [2]. The most discussed $e^{+} e^{-} \rightarrow W W$ process in this context has a theoretical drawback as it involves two $W W Z$ and $W W \gamma$ couplings intertwined, leading inevitably to a model-dependent analysis. In our study we will concentrate on the phenomenology of a single $W W \gamma$ coupling. In [2, 3] it was argued that the

$$
e^{+} e^{-} \rightarrow \nu \bar{\nu} \gamma
$$

channel is a good candidate for such measurements, because the process is sensitive only to this coupling, is of clean hadron-free signature and has a rather large cross section. Such final states have been used at PETRA, SLAC and LEP1 «1 as a means of measuring the number of light neutrinos since, at these low energies, the contribution from $W W \gamma$ coupling is negligible. However, at higher energies the $t$-channel $W$-exchange process becomes important, allowing for a direct study of $W W \gamma$ coupling, independently of the $W W Z$ coupling. Therefore it opens an exciting possibility since new physics can, and in general will, manifest itself in corrections to the gauge-boson sector, in particular in the $W W \gamma$ vertex.

The events with photon(s) plus missing energy in $e^{+} e^{-}$collisions might originate also from other mechanisms, signalling new physics beyond the Standard Model. For example, such final states can be produced in both gravity- and gauge-mediated supersymmetric models. The missing energy in these events is caused by weakly interacting supersymmetric particles, such as gravitinos, neutralinos and/or sneutrinos [5]. In all such cases the Standard Model $e^{+} e^{-} \rightarrow \nu \bar{\nu} \gamma$ events are irreducible background and reliable predictions for them are therefore necessary.

Recently the process $e^{+} e^{-} \rightarrow \nu \bar{\nu} \gamma$ has been exploited by the ALEPH collaboration to derive preliminary limits on anomalous $W W \gamma$ couplings from data collected at 161, 172 and $183 \mathrm{GeV}$ [6]. Although the results for the couplings $\Delta \kappa_{\gamma}=0.05_{-1.1}^{+1.2}$ (stat) and $\lambda_{\gamma}=-0.05_{-1.5}^{+1.6}$ (stat) are not competitive with the ones expected to be derived (in a model-dependent way) from $W W$ final states at these energies, they demonstrated the 
physics potential of processes with isolated photons. Process (1) has also been used in searches for supersymmetric signals [7].

To draw any conclusions on new physics from the experimental data, however, a proper treatment of higher-order QED corrections to the process in eq. (1) is required. In the present paper we will discuss a Monte Carlo program that can be useful in reaching such a goal. As a specific example we consider the $\nu \bar{\nu} \gamma$ final states as a means to investigate the $W W \gamma$ coupling. Our paper is organized as follows. First we will present basic distributions obtained from single bremsstrahlung analysis prior to this work and recall that they were shown to be sensitive to anomalous $W W \gamma$ couplings. We will also show that they are prone to higher-order QED corrections. Then we will present the KORALZ Monte Carlo program in which a realistic algorithm of how to match the single-photon matrix element for $e^{+} e^{-} \rightarrow \nu \bar{\nu} \gamma$ (including anomalous $W W \gamma$ coupling) with higher-order QED corrections has been implemented. We will present consistency tests of such an approach. Finally we will turn to the question of the choice of physical quantities that are most sensitive to anomalous couplings when higher-order QED corrections are switched on. Discussion of numerical results will conclude the paper.

\section{2. $W W \gamma$ vertex and higher-order QED corrections}

Expressed in purely phenomenological terms, the effective Lagrangian for the $W W \gamma$ interaction can be written in terms of seven anomalous form factors [8]. Assuming $C$ and $P$ conservation' reduces their number to three, which are conventionally denoted by $\Delta g_{1}^{\gamma}$, $\Delta \kappa_{\gamma}$ and $\lambda_{\gamma}$. The effective Lagrangian thus restricted can be written as follows

$$
\begin{aligned}
\mathcal{L}_{\text {eff }}^{W W \gamma}= & -i e\left[\left(1+\Delta g_{1}^{\gamma}\right)\left(W_{\alpha \beta}^{\dagger} W^{\alpha}-W^{\dagger \alpha} W_{\alpha \beta}\right) A^{\beta}+\left(1+\Delta \kappa_{\gamma}\right) W_{\alpha}^{\dagger} W_{\beta} A^{\alpha \beta}\right. \\
& \left.+\frac{\lambda_{\gamma}}{M_{W}^{2}} W_{\alpha \beta}^{\dagger} W^{\beta}{ }_{\sigma} A^{\sigma \alpha}\right]
\end{aligned}
$$

where $A_{\alpha \beta}=\partial_{\alpha} A_{\beta}-\partial_{\beta} A_{\alpha}$ and $W_{\alpha \beta}=\partial_{\alpha} W_{\beta}-\partial_{\beta} W_{\alpha}$. All higher derivative terms can be absorbed by the above couplings, provided they are treated as form factors and not constants.

For the photons on-shell, as is the case in the process of interest $e^{+} e^{-} \rightarrow \nu \bar{\nu} \gamma$, electromagnetic gauge invariance requires that $\Delta g_{1}^{\gamma}\left(q^{2}=0\right)=0$. Therefore we are left with only two unknown form factors. In the Standard Model both couplings $\Delta \kappa_{\gamma}$ and $\lambda_{\gamma}$ vanish.

In the process $e^{+} e^{-} \rightarrow \nu \bar{\nu} \gamma$, there are only two kinematic measurable variables at our disposal, for example the energy and the polar angle of the observed photon. In order to achieve the best sensitivity to anomalous couplings, it was shown in Refs. [6, 9] that double-differential distributions of photons measured in the barrel detector are best suited. However, for the discussion of our procedure it will be sufficient to refer to one-dimensional differential distributions.

\footnotetext{
${ }^{1}$ Subject to possible cancellations between various contributions, data on the neutron electric dipole moment constrain these form factors very severely.
} 
We use the following set of selection criteria, which have been used by ALEPH: (i) photon transverse momentum $p_{T}>0.05 \sqrt{s}$, (ii) photon energy $E_{\gamma}>0.1 \sqrt{s}$, (iii) photon angular acceptance region $\left|\cos \theta_{\gamma}\right|<0.95$, and for definiteness we take the centre-of-mass energy $\sqrt{s}=172 \mathrm{GeV}$. In Fig. 1 the energy, the angular and the transverse momentum distributions for a single photon are shown for three cases: genuine $\mathcal{O}(\alpha)$ SM results (solid lines), SM including multiphoton radiation but with $W W \gamma$ interaction exluded (stars), and genuine $\mathcal{O}(\alpha)$ including anomalous $W W \gamma$ coupling (open circles). The maximum around $E_{r}=60 \mathrm{GeV}$ in the energy distribution corresponds to the so-called radiative return to the $Z$, i.e. the photon energy for which the invariant mass of the recoiling system $\nu \bar{\nu}$ pair is close to the $Z$ mass.

There are several comments to be made. Comparing solid lines with stars, it is seen that photons with energies higher than $E_{r}$ are best suited for the $W W \gamma$ coupling measurement, because of a sizeable sensitivity to anomalous contributions. For the energies matching radiative return, the significance is very small because of the high Standard Model background. For energies below $E_{r}$ the photons are also sensitive to anomalous couplings, although the sensitivity decreases systematically with decreasing photon energy. Below $20 \mathrm{GeV}$ the sensitivity is negligible even to rather strong couplings, which we have taken in the calculations, namely $\Delta \kappa_{\gamma}=-10, \lambda_{\gamma}=0$. We will use this set of anomalous couplings throughout the paper as an example. The above conclusions are derived at the single-photon emission level. This might be sufficient to estimate the discovery power of the appropriate observables. However, it must be completed with a careful analysis of higher-order QED corrections because these may change not only the size but also the shape of the distributions that are analysed. To illustrate this point, in Fig. 11 we include for comparison the results of a multiphoton higher-order Monte Carlo simulation performed with the help of the KORALZ program [10], in which the $W W \gamma$ coupling is not included (open circles). For photons of energies higher than that of radiative return, the effect of higher-order QED corrections is small; they slightly decrease the SM cross section. In that kinematical region the inclusion of higher-order correction does not seem to be essential for our choice of the anomalous couplings. The situation is different for photon energies below that for radiative return. Contributions from both anomalous couplings and higher-order QED corrections to the distributions are of similar order and have the same sign (for the values of $\Delta \kappa_{\gamma}$ and $\lambda_{\gamma}$ chosen). Higher-order corrections vary in that region from $-30 \%$ to about $90 \%$. This result shows that any realistic determination of the anomalous $W W \gamma$ coupling must compare data with theoretical predictions that include anomalous terms as well as higher-order QED corrections at the same time. This observation is the main motivation for our present work.

\section{Monte Carlo generator}

The most up-to-date published description of the KORALZ 4.02 program for production of pairs of leptons, including radiative QED corrections, is described in detail in Ref. [10]. Real photon radiation can be generated at different levels of sophistication. 

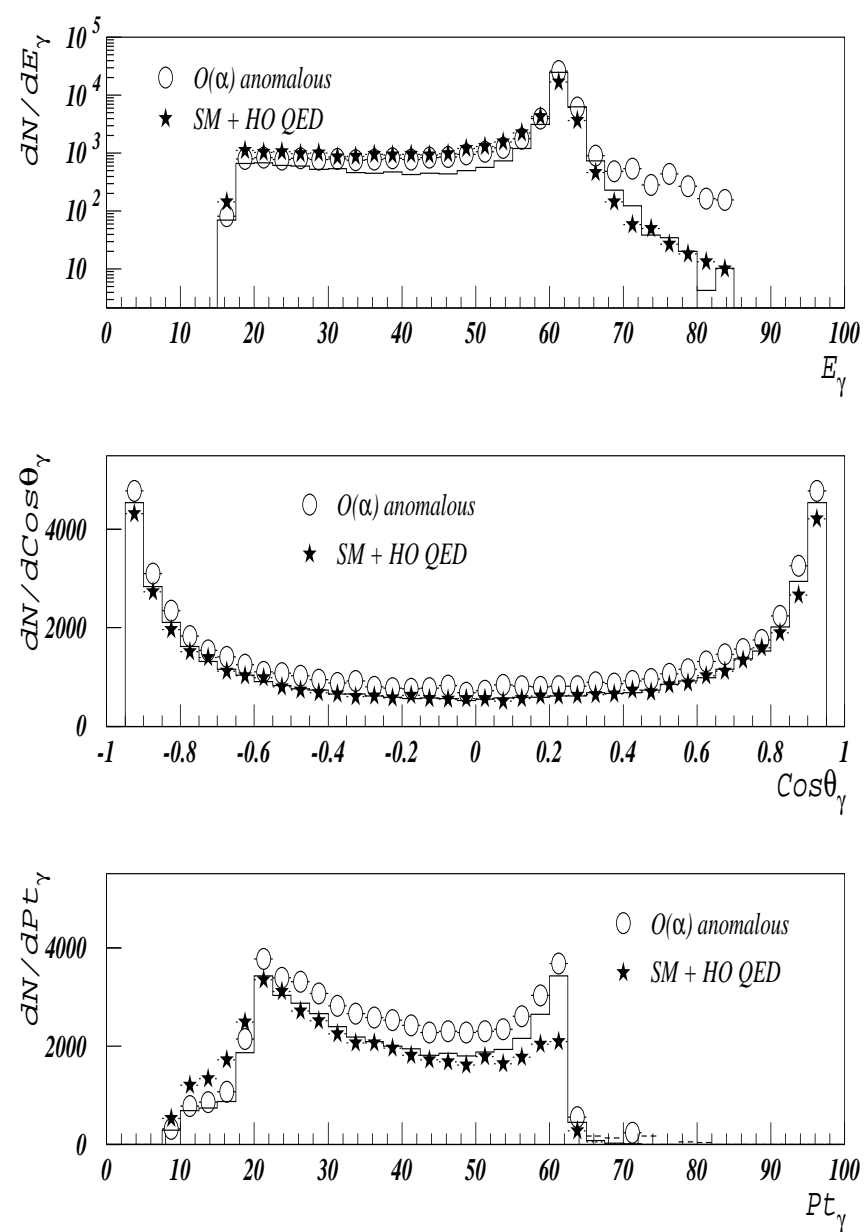

Figure 1: Inclusive photon distributions for: (i) genuine order- $\alpha$ distributions in the Standard Model - solid line; (ii) order- $\alpha$ including anomalous interactions - open circles; and (iii) the SM with multiphoton radiation - no $W W \gamma$ interaction - stars. For the set of experimental cuts and anomalous couplings see the text.

First, the program may be run at the Born level for the $e^{+} e^{-} \rightarrow l \bar{l}$ process ${ }^{2}$. Second, it may be run at order $\alpha$ when single bremsstrahlung configurations are generated. Finally, the program can be run with pragmatic order- $\alpha^{2}$ QED corrections including exclusive exponentiation. In this case the number of photons actually generated is not restricted.

Let us briefly recall the main features of KORALZ existing prior to the present work, which are relevant for $e^{+} e^{-} \rightarrow \nu \bar{\nu} \gamma$ production. The basic design of KORALZ is for the $s$-channel processes, such as muon or tau lepton pair production. The cases of muon and tau neutrinos accompanied by a single photon, see diagrams (1) and (2) in Fig. 2, have been implemented easily, since the modification is straightforward, consisting of changing the appropriate coupling constants only. In the case of electron neutrinos, there are additional contributions from $t$-channel $W$ exchange, diagrams (3) and (4), and from the $W W \gamma$ vertex, diagram (5) in Fig. 2. Since in physical gauge the amplitude for the diagram (5) with $W W \gamma$ vertex is finite in soft and collinear limits and negligible at LEP1 [11], it was not implemented at the time. Note that by neglecting the $W W \gamma$ vertex the generated distributions are formally not gauge-invariant. Diagrams (3) and (4) have

\footnotetext{
${ }^{2}$ This option is not interesting for $l=\nu$ since no photon radiation is present.
} 


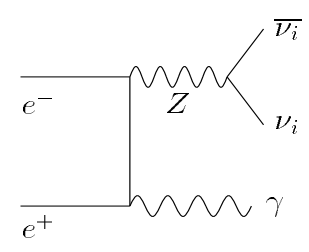

(1)

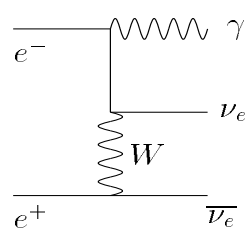

(3)

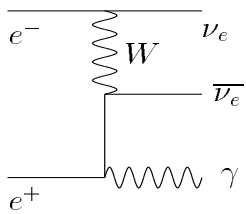

(4)

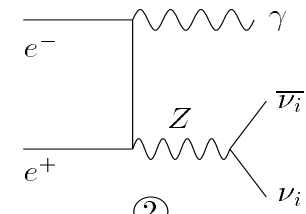

(2)

Figure 2: The Feynman diagrams for $e^{+} e^{-} \rightarrow \bar{\nu} \nu \gamma$.

been implemented in an approximate way ${ }^{\text {[ }}$ as follows. First a series of (weighted) events is generated inside KORALZ according to the Born-level $s$-channel $Z$-exchange diagrams for neutrino pair production (leading to totally unobservable final states), and with higherorder QED corrections including additional virtual and/or real photons. In this way final states consisting of $0,1,2,3, \ldots$, photons accompanying the neutrino pair are generated according to the prescription of exclusive exponentiation of YFS [12. Full phase space is covered by the generation. At this point the complete event with four momenta of final-state neutrinos and photons is constructed and stored in the common block. This procedure is correct for $\nu_{\mu} \bar{\nu}_{\mu} n \gamma$ final states. For the electron neutrinos, the matrix element for the hard process $e^{+} e^{-} \rightarrow \nu_{\mu} \bar{\nu}_{\mu}$ (with $Z$ exchange only) has to be replaced by the matrix element for $e^{+} e^{-} \rightarrow \nu_{e} \bar{\nu}_{e}$ (with $Z$ and $W$ exchanges). Consider first $\mathcal{O}(\alpha)$ contributions coming from $Z(1+2)$ and $W(3+4)$ diagrams. Thanks to the factorization properties of the QED, the matrix element squared $\left|\mathcal{M}_{0}\right|^{2}$ for the sum of four diagrams $(1+2+3+4)$ is approximated as a product of the matrix element squared $\left|\mathcal{M}_{12}\right|^{2}$ for two diagrams $(1+2)$ and the weight factor $F_{W}$ :

$$
\begin{aligned}
\left|\mathcal{M}_{0}\right|^{2} & \simeq\left|\mathcal{M}_{0}^{\text {approx }}\right|^{2}=\left|\mathcal{M}_{12}\right|^{2} F_{W}, \\
F_{W} & =\frac{\left|\mathcal{M}_{Z}+\mathcal{M}_{W}\right|^{2}}{\left|\mathcal{M}_{Z}\right|^{2}},
\end{aligned}
$$

where $\mathcal{M}_{Z}$ and $\mathcal{M}_{W}$ denote amplitudes for $\nu_{e} \bar{\nu}_{e}$ pair production via $Z$ - and $W$-exchange diagrams, respectively, with no photons in the final state. In the calculation of the factor $F_{W}$ four momenta of $\nu_{e}$ and $\bar{\nu}_{e}$ are used in a straightforward way, but for the incoming electrons and positrons effective beam momenta are calculated from momenta of the real beams and real photon emitted, according to the prescription given in Refs. [10, 13].

\footnotetext{
${ }^{3}$ It is thus important to realize that the program quality for $\nu_{e} \bar{\nu}_{e} \gamma$ final state was (and remains) inferior to that for other final states.
} 
Note that the factor $F_{W}$ given in eq. (3) is gauge-invariant. The standard final rejection of KORALZ creates the sample of unweighted events, which is passed to the program user. The factor $F_{W}$ is included in that final rejection weight.

Let us stress that in this way the effect of diagrams (3) and (4) with $t$-channel $W$ exchange, as well as their interference with the diagrams (1) and (2), has been introduced not only for case of a single-photon radiation, but also for multiphoton configurations. This approximation, as well as the whole program, has been heavily tested at LEP1 energies both for the single bremsstrahlung mode and the multiple photon case. Extensive comparisons with the results from another program [14], including a complete matrix element for single and double bremsstrahlung, have been performed, finding an excellent agreement and thus proving both the validity of the approximation and the correctness of the code, see Refs. [10, 13, 15] for more details. During the last seven years, i.e. since the $\nu \bar{\nu}$ pair production process has been implemented into KORALZ for the first time [15], the program was modified several times, and in general its applicability for $s$-channel processes was assured also at LEP2 energies, see [16].

For the case $\nu_{e} \bar{\nu}_{e} \gamma$, however, the $W W \gamma$ interaction (and the question of gauge invariance) can no longer be ignored, and the approximate treatment of $t$-channel $W$ exchange requires refinements to be precise enough at LEP2 energies.

We now turn to the discussion of how these problems are solved in the present version 4.04, which is the modification of the version 4.02 of the KORALZ program. The modification consists of introducing the new weight defined as a ratio of matrix element squared for $e^{+} e^{-} \rightarrow \nu \bar{\nu} \gamma$ with and without the $W W \gamma$ vertex:f

$$
W_{T}=\frac{\left|\mathcal{M}_{0}+\mathcal{M}_{W W \gamma}\right|^{2}}{\left|\mathcal{M}_{0}\right|^{2}} \text {. }
$$

The term $\mathcal{M}_{W W \gamma}$ contains contributions from diagram (5) with the $W W \gamma$ vertex, including anomalous couplings. The matrix element is taken from the numerical program developed in Ref. [9]. Note that the term in the denominator $\left|\mathcal{M}_{0}\right|^{2}$ is gauge dependent. If in the present version KORALZ 4.04 the events were generated according to the matrix element $\left|\mathcal{M}_{0}\right|^{2}$, the gauge dependence would cancel out in the product of eqs. (3) and (1). However, this is not the case, since they are generated according to the approximate $\left|\mathcal{M}_{0}^{\text {approx }}\right|^{2}$ as explained above. Nevertheless, as we will discuss in the next chapter, see Fig. 3, the numerical error is small and the final distributions are affected by this approximation in a small and controlled manner only. The implementation of the above weight $W_{T}$ proceeds as follows. For every event, the complete kinematic configuration is taken. In cases when there is just one photon, the formula (4) can be used in a direct way. However, for events with multiple photons generated, there are many possibilities for a choice of a photon radiated off the $W$ boson. Since the QED bremsstrahlung of high- $p_{T}$

\footnotetext{
${ }^{4}$ In some regions of phase space, due to destructive interference of contributions from the $s$ - and $t$ channel boson exchanges, the Standard Model predictions can be very small. There the weight defined in eq. (4) can in principle tend to infinity, making the simulation numerically non-convergent. This technical obstacle, not relevant to our discussion, is overcome by replacing the SM matrix element by a different one and obtaining the SM predictions also in the form of a weighted event sample.
} 

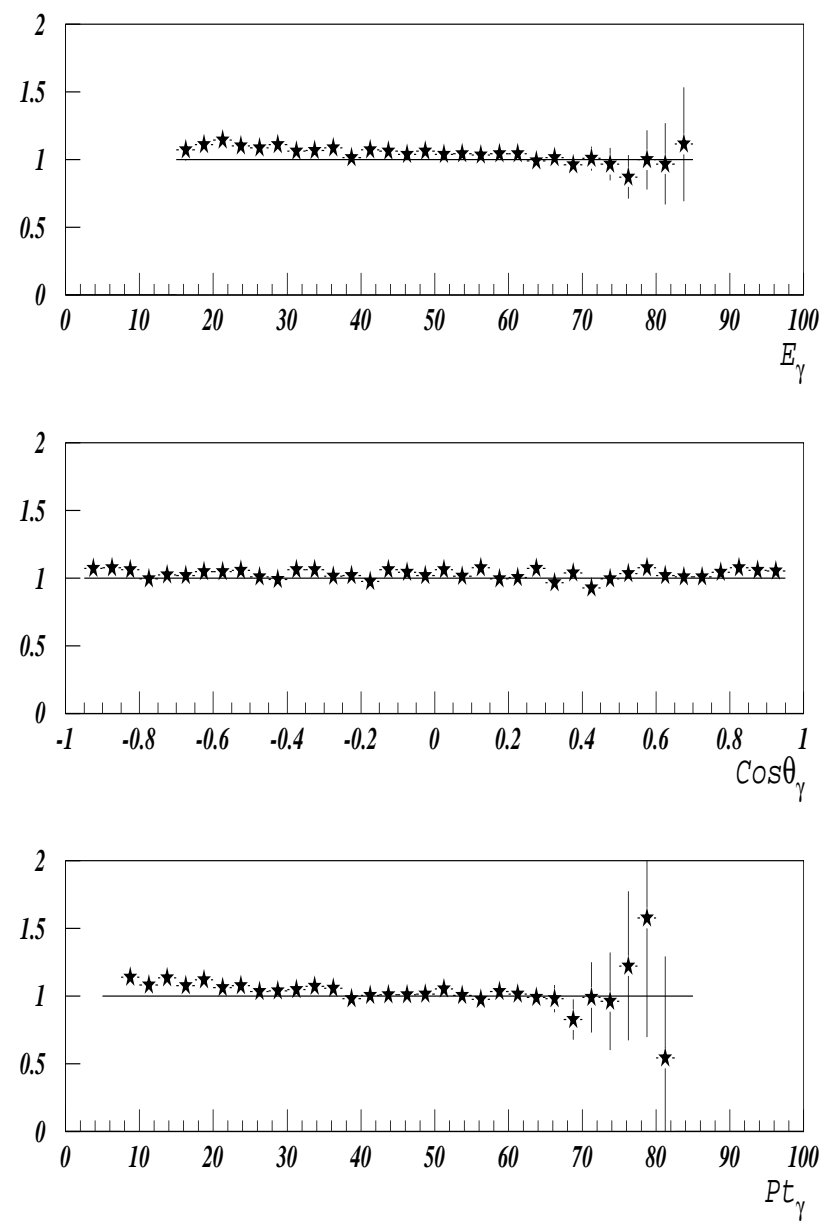

Figure 3: The ratio of distributions generated by the KORALZ 4.04 and the program used in Ref. [9]. Both programs are run with the same input parameters and kinematical cuts. Anomalous $W W \gamma$ couplings are included.

photons from electrons is strongly suppressed, and the $W W \gamma$ contribution is important for energetic photons, in our algorithm we calculate f the weight factor $W_{T}$ in eq. (田) only for the photon of highest $p_{T}$. All remaining photons are then incorporated into effective beams according to the following recipe:

a) Except for the photon of highest $p_{T}$, all remaining photon's four-momenta are subtracted from the four-momentum of the beam of the same hemisphere. In this way we obtain effective electron beams, which are not necessarily on-mass-shell.

b) We boost all four-momenta of effective beams, neutrinos as well as the highest- $p_{T}$ photon to the rest frame of effective beams.

c) We reset the four-momenta of the effective electron beams to be on-mass-shell in such a way that their back-to-back direction and energy-momentum conservation is preserved.

At this point we have constructed reduced on-mass-shell kinematics for $e^{+} e^{-} \rightarrow \nu \bar{\nu} \gamma$ configuration for which the weight $W_{T}$ can be calculated according to eq. (4)). For practical reasons explained at the end of the next chapter, we do not include the $W_{T}$ weight into

\footnotetext{
${ }^{5}$ At this point we assume that the $W W \gamma$ couplings do not affect the matrix elements in soft or collinear photon limits, or virtual corrections. In short, it means that the whole structure of QED remains intact and that the sole effect of new couplings is a modification of the amplitude for configurations with photons of high energy and $p_{T}$.
} 
KORALZ internal rejection loop. Instead, generated sample follows the same distributions as in previous versions of the program. The $W W \gamma$ contribution manifests itself only through the weight $W_{T}$ which is provided for every event.

\section{Numerical tests}

The reduction procedure presented above is not free of systematic uncertainties for $W W \gamma$ contributions to multiphoton production processes, except at the single photon level. These uncertainties, though, are beyond the leading-log QED level as well as beyond soft-photon corrections. Only genuine next-to-leading-log corrections to contributions from $W W \gamma$ interactions are out of control. On the other hand, one benefits from full coverage of the bremsstrahlung photons phase space provided by the host Monte Carlo as well as its predictions for Standard Model without any deterioration in quality.

As a first step in testing the implementation of the $W W \gamma$ vertex (with or without anomalous couplings) and diagrams including $t$-channel $W$-exchange into KORALZ, we performed studies at a single-photon level. In this case results from KORALZ should be the same as from direct Monte Carlo integration (using weighted event sample), as e.g. in the original approach of [9]. Any discrepancy would indicate either technical problems in any of the two programs, or breakdown of our approach. The results are presented in Fig. 3, where the ratios of differential cross sections obtained from two programs are shown. The results are consistent over the entire phase space ${ }^{\text {D }}$. One can see that distributions differ by at most a few percent. This also shows that the numerical error due to breaking the gauge invariance by our procedure is small.

In the second step we verify that the reduction procedure to effective beams as explained in the previous section indeed works. For that purpose we compare at a given cms (center-of-mass system) energy the results from KORALZ with anomalous contributions and higher-order corrections included as described above, with another approach (called EMU), where convolution of the $W W \gamma$ amplitude with higher-order corrections is performed as explained below. In the EMU approach, we first generate the spectrum of $M_{\text {eff }}$ from the multiphoton run of KORALZ at cms energy without any $W W \gamma$ interaction. The

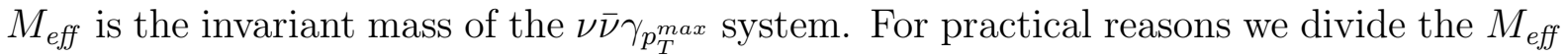
spectrum into discrete bins. In the second step, for each bin $i$ containing $N_{i}$ events we generate an equal number of events, this time, however, at "an effective cms energy" equal to the centre of the bin $M_{\text {eff }}$ with the help of a single bremsstrahlung mode of KORALZ operation and contributions from the $W W \gamma$ interaction included (which we have shown before to work properly). In this case additional photons are not present, but we emulate their presence by a boost of all final-state particles from the "effective cms energy" $M_{\text {eff }}$ to the overall cms frame as if there was (just one) additional photon collinear to one of the beams.

As can be seen in Fig. 田, the two plots differ in the physically interesting region! by

\footnotetext{
${ }^{6}$ For large $E_{\gamma}$ or $p_{T}^{\gamma}$ the cross section is very small. Large statistical fluctuations in the ratios of the Monte Carlo results are thus expected.

${ }^{7}$ Physically interesting region consists in this case of photons of $p_{T}>20 \mathrm{GeV}$ and of energies different from radiative return to the $Z$.
} 

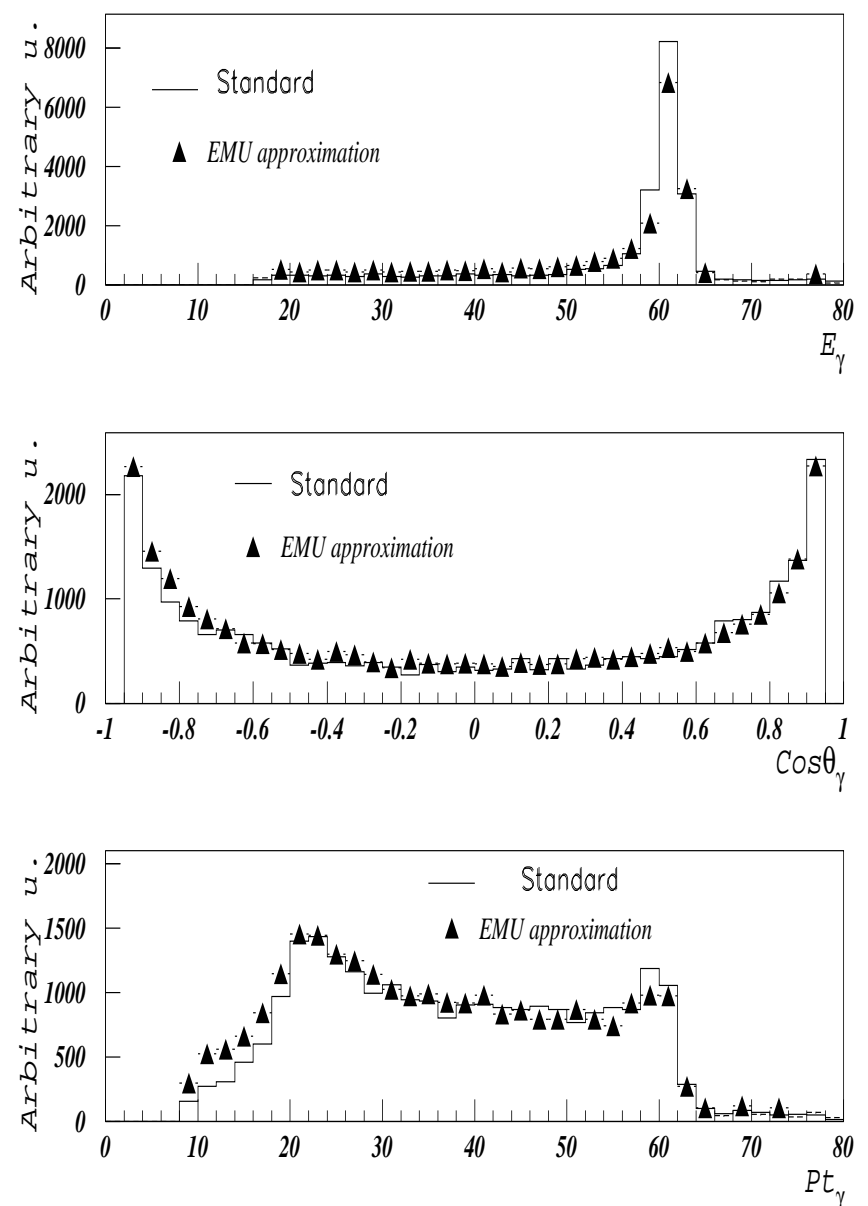

Figure 4: Comparison of differential distributions obtained from the new version of KORALZ 4.04 including anomalous interactions (Standard), and the EMU algorithm (triangles), as explained in the text.

only up to a few per cent. This indicates that the proposed algorithm is constructed properly and also that the bulk of the higher-order corrections is of leading-log type.

One should bear in mind that such tests are indicative only. To answer the question of how good such an algorithm is, would require careful comparisons of the generated distributions with those from exact matrix-element calculations including anomalous couplings of order at least $\alpha^{2}$. This is difficult and not necessary at the moment, as a precision on higher-order corrections to anomalous contributions of order $10 \%$ is sufficient.

In practical applications it is convenient to use the method of correlated samples. The $W W \gamma$ vertex is linear in $\Delta \kappa_{\gamma}$ and $\lambda_{\gamma}$, the two independent anomalous couplings. Therefore the matrix element squared, and consequently all differential distributions, are bilinear in the variables $\Delta \kappa_{\gamma}$ and $\lambda_{\gamma}$. It is thus convenient to calculate, for every generated point in phase-space, the value of the matrix element at six fixed and conveniently chosen combinations of $\Delta \kappa_{\gamma}$ and $\lambda_{\gamma}$ values 8 . From this, the matrix element for any other value of anomalous couplings can be easily recalculated. This property extends to differential distributions as well. It also remains valid when higher-order leading-log QED corrections as explained above are included.

${ }^{8}$ In KORALZ numerical values of $\left(\Delta \kappa_{\gamma}, \lambda_{\gamma}\right)$ actually implemented are: $(0,0),(-10,0),(10,0),(0$, $-10),(0,10),(10,10)$. 
Note that KORALZ generates unweighted events for the Standard Model with additional weights corresponding to different modifications of the matrix element due to $W W \gamma$ couplings. This not only saves computing time, as it is sufficient to perform detector simulation only once, but also the standard and non-standard model samples obtained this way are statistically correlated, as was explained in [17]. This significantly improves the convergence of the predictions since the statistical error affects only the difference of the two distributions.

After these modifications, KORALZ version 4.04 (or higher) can be usedff for our purpose with any combination of flags for the initial state. Every event generated as weight-one event, corresponding to the Standard Model with $W W \gamma$ vertex excluded $\square$, is accompanied now by the weight record, allowing for a calculation of weights for any numerical value of $W W \gamma$ coupling, standard or anomalous. This can be especially useful in performing final fits to the data in the presence of experimental cuts. It can also be used for Standard Model background calculations for other processes with a single photon plus missing energy in the final state.

Finally, let us note that the question of systematic errors of the genuine $s$-channel process for non-electron neutrino pair production was not addressed. It calls for a separate discussion including tests/comparisons with, for instance, the matrix element for the production of a neutrino pair with up to three real protons.

\section{Results and Conclusions}

Let us now turn to the presentation of the numerical results for anomalous couplings. In Fig. 5 we compare the photon spectra normalized to the SM lowest-order calculations for three cases: (i) anomalous couplings are included at the lowest order (circles), (ii) higher-order QED corrections but no anomalous couplings are included (stars), and (iii) both anomalous couplings and higher-order corrections are included (triangles). The difference of physical content between this plot and the one of Fig. 11 consists primarily in presenting the results for the case where higher-order radiative corrections and anomalous contributions are included simultaneously. Although the QED higher-order bremsstrahlung corrections are sizeable, and varying from $-30 \%$ to $100 \%$, they do not wash out the effect of the anomalous contribution. What is more interesting, in the region of high-energy photons the presence of higher-order effects tends to slightly enhance the sensitivity to anomalous couplings. On the other hand, for photon energies below that of radiative return, the sensitivity deteriorates significantly. We also observe that the combined effect of anomalous couplings and radiative corrections is not just a simple sum of the two effects.

We have thus shown that the interpretation of experimental data for anomalous $W W \gamma$ couplings in the $e^{+} e^{-} \rightarrow \nu \bar{\nu} \gamma$ channel requires theoretical predictions in the form of a Monte Carlo simulation program, which includes anomalous contributions as well as

\footnotetext{
${ }^{9}$ The version 4.04 can be obtained upon request from Zbigniew.Was@cern.ch. It includes also options described in 18.

${ }^{10}$ Exactly as in the versions of the program prior to the modifications presented here.
} 

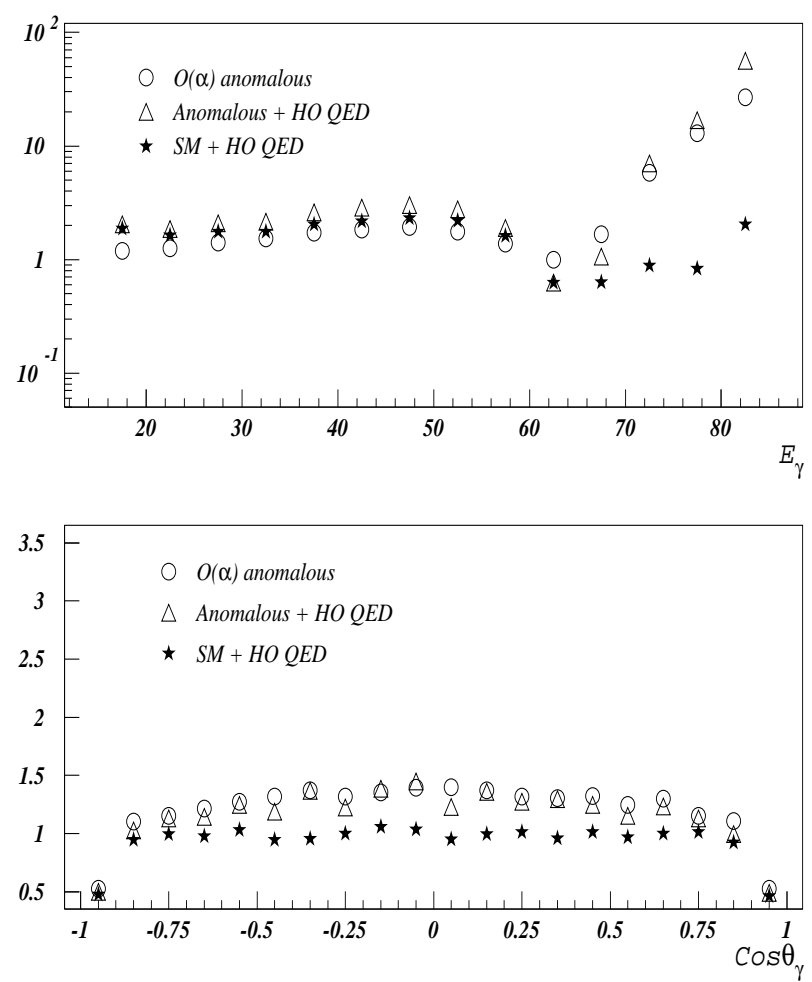

Figure 5: The ratios of $E_{\gamma}$ and $\cos \theta_{\gamma}$ distributions obtained from: (i) order- $\alpha$ distributions including anomalous couplings - open circles, (ii) multiple photon radiation, no anomalous couplings - stars, (iii) multiple photon radiation and anomalous couplings included triangles. In all cases distributions are divided by the appropriate ones obtained from the single-photon generation in the Standard Model (no anomalous couplings). All input parameters as explained in the text.

multiple bremsstrahlung higher-order QED corrections. The current version 4.04 of the KORALZ program, with modifications presented in this paper, can now be used to achieve this goal. It can also be used for Standard Model background calculations for other interesting processes, such as gravitino or neutralino/sneutrino production processes at LEP2.

\section{Acknowledgements}

This work has been supported in part by the Polish-French Collaboration within IN2P3 (AJ), by the Polish State Committee for Scientific Research grant 2 P03B 03014 (JK), and by the II Maria Skłodowska-Curie Fund PAA/DOE-97-316 (ZW). ZW acknowledges the hospitality and support of the ALEPH Collaboration group in LAL Orsay. JK thanks the CERN Theory Division for hospitality during the final stage of this work. 


\section{References}

[1] D. Bardin et al., hep-ph/9709270, in Proc. "Physics at LEP2", eds. G. Altarelli, T. Sjöstrand and F. Zwirner (CERN 96-01, Geneva, 1996) Vol. 2, p.No. 3

[2] G. Gounaris et al., hep-ph/9601233, ibid., Vol. 1, p.No. 525

[3] G. Borisov, V.N. Larin and F.F. Tikhonin, Z. Phys. C41 (1988) 287;

K.J. Abraham, J. Kalinowski and P. Ściepko, Phys. Lett. B339 (1994) 136

[4] E. Ma and J. Okada, Phys. Rev. Lett. 41 (1978) 287;

K.J.F. Gaemers, R. Gastmans and F.M. Renard, Phys. Rev. D19 (1979) 1605;

G. Barbiellini, B. Richter and J.L. Siegrist, Phys. Lett. B106 (1981) 414

[5] P. Fayet, Phys. Lett. B117 (1982) 460;

J.A. Grifols, X. Mor-Mur and J. Solà, Phys. Lett. B114 (1982) 35;

for recent discussions of these processes, see for example A. Brignole, F. Feruglio and F. Zwirner, hep-ph/9711516, and G.I. Kane, hep-ph/9709318, talk given at SUSY97, Philadelphia, and references therein

[6] ALEPH Collab., Isolated photons and constraints on anomalous $W W \gamma$ couplings at LEP2, preprint EPS-HEP/97-599, also ALEPH 98-012/CONF 98-002;

[7] R. Barate et al., ALEPH Collab., Searches for Supersymmetry in the photon(s) plus missing energy channels at $\sqrt{s}=161$ GeV and 172 GeV, preprint CERN-PPE/97122

[8] K. Hagiwara et al., Nucl. Phys. B282 (1987) 253

[9] D. Choudhury and J. Kalinowski, Nucl. Phys. B491 (1997) 129

[10] KORALZ version 4.0, S. Jadach, B.F.L. Ward and Z. Wa̧s, Comput. Phys. Commun. 79 (1994) 503

[11] F.A. Berends et al., Nucl. Phys. B301 (1988) 583

[12] For a pedagogical introduction, see for example:

S. Jadach and B.F.L. Ward, preprint CERN-TH.5399, in Proc. "Radiative Corrections for $e^{+} e^{-}$Collisions", ed. J.H. Kuhn (Springer-Verlag, 1989) p.No. 118;

Z. Wass, "Radiative Corrections", preprint CERN-TH.7154, in Proc. 1993 European School of High Energy Physics, eds. N. Ellis and M.B. Gavela (CERN 94-01, Geneva, 1994) p.No. 307

[13] P. Colas, R. Mirabito and Z. Wa̧s, Munich Preprint MPI PAE-Exp El. 211 (1989)

[14] M. Martinez, R. Miquel and C. Mana Proc. "Radiative Corrections: Results and Perspectives", eds. N. Dombey and F. Boudjema (Plenum Press, 1990) p.No. 578 R. Miquel, C. Mana and M. Martinez, Z. Phys. C48 (1990) 309 
[15] P. Colas, R. Miquel and Z. Wąs, Phys. Lett. B246 (1990) 541

[16] KORALZ version 4.02, in F. Boudjema et al., in the same Proc. as Ref. [1], Vol. 1, p.No. 207

[17] S. Jadach and Z. Wa̧s, Phys. Rev. D41 (1990) 1425

[18] T. Paul and Z. Wạs, Inclusion of $\tau$ Anomalous Magnetic and Electric Dipole Moments in the KORALZ Monte Carlo, L3 Internal Note 2184 (1997), http://13www.cern.ch/note/notes1997.html and hep-ph/9801301 